\title{
Ueber einen epithelialen Tumor des Ciliarkörpers.
}

\author{
Von \\ Dr. R. Schlipp \\ ans Wiesbaden. \\ Hierzu Tafel XV, Figur 1 und 2.
}

(Aus der k. k. Universitäts-Augenklinik des Herrn Prof. Fuchs in Wien).

Die ophthalmologische Literatur berichtet nur sechs Mal über epitheliale Geschwülste des Ciliarkörpers; erscheint hierdurch allein schon jeder neue Fall dieser seltenen Gattung der allgemeineren Keuntniss werth, selbst. wenn die Untersuchung desselben nur bereits bekannte anatomische Eigenschaften bestätigte, so glaube ich die Beschreibung des nachfolgenden Falles um so mehr rechtfertigen zu können, als ihn ganz besondere anatomische Eigenthümlichkeiten vor den bis heute bekannt gewordenen epithelialen Tumoren des Ciliarkörpers auszeichnen.

Unsere Beobachtung betrifft das rechte Auge eines zehnjährigen Mädchens, welehes am 26. März 1890 von ihren Eltern auf die Klinik gebracht wurde, mit der Angabe, dass das Ange seit zwei Jahren'bereits erblindet sei, ohne dass irgend eine Ursache hierfitr zu eruiren wäre.

Die Untersuchung zeigte die Lider normal, die Conjunetiva auffallend blass. Der Bulbus war stark geschrumpft und die Spannung: desselben heralgesetzt; die Cornea erschien matt, aus der Pupille ein gelber Reflex. Die Iris war stark geschrumpft. Die Patientin litt nie an Schmerzen. Diagnose: Atrophï bulbi dextr. Das linke Auge war normal.

Am 27. III. 1890 wurde die Enucleation vorgenommen und Patientin konnte schon am 5. IV. wieder entlassen werden. Die 
Patientin befindet sich heute, also neun Jahre nach der Enueleation, vollkommen woll.

Die mikroskopische Untersuchung des Bulbus, welcher in Mïller'seher Flỉssigkeit gehärtet war, ergiebt Folgendes:

Das Epithel der Cornea erscheint im Ganzen niedrig, an einzelnen Stellen nur aus zwei Schichten bestehend; es sind vielfach oberflächliche Zellen ausgefallen, so dass kleine Lücken entstehen, welche dem matten Aussehen der Hornhaut im Leben entsprechen. Eine normale Bowman'sche Membran ist nur in den centralen Theilen erhalten, wäluend dieselbe in den Randtheilen durch eine Gewebsschichte ersetzt ist, die eine feine, etwas unregelmässige Faserung besitzt und kerm- und gefässreich erseheint. Das Parenchym zeigt einzelne Biutextravasate nud ist von zahlreichen tiefen Gefässen durchsetzt, welche in den Schnitten zum Theil quer, zum Theil längs getroften sind und ron Leukocyten eingescheidet werden. Gegen die nasale Seite hin ist die Bowman'sche Membran eine Strecke weit von einer faserigen Schichte uberlagert, welcher an der Hinterwand der Cornea ein Vorsprung von festem, straffem Gewebe entspricht, welcher von vielfach gefalteter Descemet'scher Membran überzogen ist, und die vordere Kanamer in eine grössere temporale und kleinere nasale Hälfte theilt; in dem temporalen 'Theil ist die Descemetsehe Membran an einer Stelle durch aufgefaserte Lamellen der Hornhaut unterbrochen.

In dem geronnenen Kammerwasser sind zahlreiche rothe Blutkörperchen suspendirt, welche in dem unteren Kammerwinkel ein dichtes Hyphaema bilden; weisse Blutkörperchen sind nur in geringer Zall zu erkennen.

Die Iris ist auf der nasalen Seite an die Comea angedräekt, atrophisch und weiterhin von einer Exsudatschwarte überlagert, welche bis in die Gegend des temporalen Ciliarkörpers reicht; letztere wird an ilurer Vorderfäche von einer neugebildeten Glashaut überzogen. Von der temporalen Trishälfte ist nur noch Pigment zu erkennen, welebes innerhalb der bindegewebigen Schwarte regellos zerstreut liegt.

Der Ciliarkörper ist atrophisch und von zahlreichen Rundzellen durchsetzt, die Ciliarfortsätze durch bindegewebige Stränge in die Länge gezogen.

Die Linsenkapsel erseheint vielfach gefaltet und verdickt. In dem hinteren Abschnitt des Kapselsacks findet sich ein gefässreiches Bindegewebe, in welches colloïde und rerkalkte Massen eingelagert sind; in dem rorderen Abschnitt besteht das Gewebe 
aus langgestreekten Gebilden, die etwa wie quergestreifte Muskelfasern an einander liegen und von kernhaltigen Zwisehenräumen getrennt sind; sie selbst enthalten keine Kerne, zeigen vielmehr eine gleichmässige Beschaffenheit, sowie eine feinere, bräufliche Pigmentirung, und sind wohl als Schläuche von Gesehwulstzellen aufzufassen, welche hyalin degenerirt sind und deren Kerne die Färbbarkeit verloren haben; die hintere Linsenkapsel zeigt auch in der 'That an einer Stelle eine Lücke, dureh welche die Geschwulstzellen, die unten näher beschrieben werden, hineinwuchern.

Auf der nasalen Seite beginnt hinter dem Schlérmm'schen Canal zwisehen dem atrophischen Ciliarmuskel und der Sklera ein spaltförmiger, sich nach hinten verbreiternder Raum, welcher von geronnenem Exsudat und rothen und weissen Blutkörperchen angefüllt exscheint. Die Chorioidea ist im vorderen Theile atrophisch und liegt hier der Tumormasse unmittelbar an. Hinter dem Ciliarmuskel verbreitert sich der suprachorioideale Raum und wird ron der aufgefaserten Suprachorioidea durchzogen, in deren Maschen rothe und weisse Blutkörperchen, sowie Geschwulstzellen liegen. Die inneren Schichten der Chorioidea zeigen in dieser Zone dentiche Invasion des Tumors und sind stellenweise ganz gesehwunden. Weiter rückwärts sieht man an verschiedenen Stellen zwischen Sklera und Chorioidea Tumorknoten, in welche noch Chorioidealreste eingestreut erscheinen. Noch weiter hinten finden sich vor der Chorioidea Knochenlamellen, welch $\theta$ hier und da Pigment einschliessen, and ferner junges, reichlich vascularisirtes und zellig infiltrirtes Bindegewebe, das direct neben dem Opticus beginnt. - Auch auf der temporalen Seite findet man Abhebung des Ciliarmuskels und der Chorioidea, die hier ebenfalls atrophisch ist, und sowrohl Bindegewebs- wie Knochenbildung zeigt.

Sowohl auf der nasalen wie temporalen Seite zieht von der Gegend des Ciliarkörpers aus je ein Bindegewebsstrang mach hinten gegen die Chorioidealschwarte hin, welcher, sich allmählich auffasernd, zwischen den Geschwalstzellen verliert. Namentlich in dem temporal gelegenen scheinen zahlreiche Gefässe zu verlaufen, die zum Theil von Leukoeyten umschlossen sind. - Retina ist nirgends, von der Papille an, zu erkennen; die Geschwalstzellen reichen bis in die Gegend des Skleralloches. - Der Opticus ist, wie die nach Weigert und Pal gefärbten Schnitte ausweisen, rollkommen atrophisch und erseheint somit kernreich, das Bindegewebe vermehrt. 
Das Bulbusinnere ist vollkommen ausgefullt von Geselmulstzellen, welche im Allgemeinen einen grossen Protoplasmaleib mit yrossem, blassem Kern und somit das Ausseben von Epithelzellen haben; dieselben lagern ferner wie Epithelzellen an einander, ohne dass bindegewebige Fasern zwischen ihnen zu erkennen wären, an vielen Stellen so, dass die einzelnen Zellgrenzen verschwinden und man nur eine grosse. Protoplasmamasse wahmimmt, in welcher die Kerne liegen. Das Protoplasma der Zellen zeigt durchweg eine diffuse, zart brännliche Färbung.

Im einzelnen sind die Zellen freilich recht verschieden, indem sie theils dureh gegenseitige Beeinfussung des Wachsthnms, theils dureh degenerative Processe veründert werden. -- Durch erstere wird, wie auch sonst bei Epithelzellen, die Form der einzelnen Zelle modifieirt, indem sie sich den Raumverhältuissen anpasst, welche durch die Nachbarzellen gegeben sind. Indessen kommt es nie zn epithelperlartigen Sehichtungen der Zellen; dagegen findet man an manchen Stellen, dass zwischen grossen, polygonalen Zellen solche liegen, die durch Abplattung zwisehen ihren grösseren Nachbarn ganz schmal geworden sind, so dass sie aussehen wie Geritstfasern, die, ähnlich wie bei alveolären Sarkomen, die einzelnen Zellen tremnen. Doch kann kein Zweifel daruber bestehen, dass es sich hier nicht um Bindegewebsfasern handelt; denn erstens haben diese verschmälerten Zellen keine Bindegewebskerne, sondem dieselben wie die anderen Zellen md zweitens färben sie sich in van Gieson-Präparaten gleich den anderen Zellen gelb, und nicht roth wie Bindegewebe.

Die degenerativen Veränderugen der Zellen sind verschiedener Art. Sie betreften die Kerne, die häufig so vergrössert sind, dass sie das mehrfaele, sogar zehnfache Volumen der gewöhnlichen Kerne erreichen; anch der Protoplasmaleib mancher Zellen ist $z$ bedentender Grösse herangewachsen; indessen kann man solche Zellen nicht als Riesenzellen im gewöhnlichen Sinne des Wortes bezeíchnen, weil sie nur einen Kern besitzen; anch jene Stellen, wo die Zellen ohne seharfe Grenze in eine gemeinschaftlicho Protoplasmamasse zusammentiessen, unterscheiden sich von Riesenzellen durch die Anordnung der Kerne, die so in der Protoplasmamasse vertheilt sind, wie es bei an einander liegenden Zellen der Fall zo sein ptegt. Eigentliche Riesenzellen finden sich also nirgends in der Geschwulst.

Von degenerativen Veränderungen ist ferner das Auftreten zahlreicher, kleiner, stark lichtbrecheuder, runder Stellen im Protoplasma zu exwähnen; sowobl nach ihrem Ausselen, wie nach 
dem Umstande, dass sie sich nach der Pal'schen Methode selswarz färben, müssen sie als Fetttröplchen angesehen werden. Dort, wo die Zellen in grösserer Ausdehnung von solchen Fettkörnchen durehsetzt sind, erhält die Geschwulst ein durehscheinendes Aussehen (Fig. 2); indessen führt die Degeneration nicht weiter; die Kerne bleiben erlalten, und nirgends ist ein vollständiger Zerfall zu beobachten; ebenso wenig kann man käsige oder sonst nekrotische Heerde irgend welcher Art in der Geschwulst finden. Ein weiteres Zeichen der Degeneration besteht in dem Auftreten homogener Kugeln in dem Protoplasma der Zellen; dieselben färben sich nach Gram blau oder blaugrün, mit Haematoxylin-Eosin, ähnlich wie die Kerme, nur etwas mehr grünlich, nach van Gieson orangeroth und sind nach dieser Färbung und ihrem homogenen Aussehen als hyaline Kugeln zu bezeichnen, die, wenn sie klein sind, den Russel'schen Körperchen gleichen. Sie entwickein sich im Protoplasma der Zellen, wachsen, confluiren und bilden an manchen Stellen grosse, höckerige oder oft stalaktitenähnliche Gebilde.

Die Geschwulst enthält nur wenige und enge Gefässe; an einzelnen Stellen sieht man kleine Heerde von einkernigen Rundzellen eingestreut und hier und da Hohlräume, die bald grösser, bald kleiner sind und ron einer gleichmässigen Masse geronnener Flüssigkeit ansgefüllt werden.

Besondere Beachtung verdienen zwei Abschnitte der Geschwulst, einmal jener, welcher den hinteren Theil des Bulbus ausfüllt und hier zwischen Skiera und Chorioidea, sowie zwischen dieser und dem aufgelagerten Knochen liegt, und ferner der dem Ciliarkörper anliegende Theil der Geschwulst.

Was den ersteren anlangt, so liegen hier zwisehen den straffen Bündeln der chovioiditischen Sehwarte, sowie zwischen den Knochenlamellen, langgestreckte Zellschläuche, welche ganz Carcinomschläuchen ähneln, nur dass dieselben, wie schon oben erwähnt, keine Zellgrenzen erkennen lassen, sondern aus einer gleichmässigen Protoplasmamasse bestehen, in welche Kerne eingelagert sind.

An dem Ciliarkörper zeigt sich Folgendes: Regelmässig gebaute Ciliarfortsätze sind nur in selr geringer Anzahl vorhanden; an den meisten Stellen zeigt sich das dem Ciliarmuskel aufliegende Stroma (Fig. 1 a.) des Ciliarfortsatzes von Zellsträngen durehwachsen, welehe en Lumen einschliessen und mit den Quer- und Schrägschnitten der tieferen Buchten, die sich auch an normalen Ciliarfortsätzen so häufig finden, grosse Aehnlichkeit 
laben. Doch sind diese Buchten hier nicht überall ron dem doppelten Zellenbelag des normalen Ciliarkörpers ansigekleidet, vielmehr nur von einer einfacken Lage grosser, protoplasmatiseher, ungefähr eubischer Zellen, mit eingelagerten braunen Pigmentkörnchen, die indessen nicht so zahlreich sind, dass sie den Kern der Zellen verdeckten.

Ob diese Zellen der äusseren pigmentirten oder der inneren unpigmentirten Schichte des Ciliarkörperepithels entsprechen, ist nicht sicher zu entscheiden; dem Aussehen und dem Pigmentgehalte nach dürten sie von der äusseren pigmentixten Lage abstammen.

Je näher man nach der Oberfläche geht, desto mehr treten anstatt der hohlen Zellschläuche solide auf, ron denen manche nur ans einer einzigen Reihe von Zellen bestehen, die aber noch immer die charakteristische Pigmentiruag des Pigmentepithels des Ciliarkörpers autweisen.

Diese soliden Zellschläuche zeigen einen directen Uebergang. in die Zellen des Tumors (Fig. 1\%.), indem die Pigmentkörnchen ans den Zellen versehwinden und kleine Fetttröpfohen in ihnen auftreten.

An einer Stelle des Ciliarkörpers der entgegengesetzten Seite sieht man stellenweise die beiden am normalen Ciliarkörper vorhandenen Zellreihen des Pigmentepithels und hier scheinen die inneren, unpigmentirten Zellen in diejenigen der Geselwwulst uberzugehen.

Demnach möchte es scheinen, als ob die Geseliwulstzellen sowohl aus der äusseren wie inneren Zellenlage hervorgingen, was an und für sich nicht unmöglich erscheint, da ja beide Zellarten ans derselben ursprünglichen Anlage der primüren Augenblase hervorgelien.

Die entzündlichen Feränderungen, welche der Bulbus darbietet, sind wohl als secundär zu bezeichnen, indem sich die wachsende Gesehwulst, wie es häufig bei intraocularen Tunoren beobachtet wird, mit einer Iridocyclitis combinirte, welche das Auge dann der Schrmpfing entgegenführte.

Nach dem Voraufgehenden haben wir hier einen primären Tumor des Ciliarkörpers vor uns, welcher der Gruppe der epithelialen Geschwïlste angehört; indessen stehen wir $\mathrm{ab}$, ihn direct als Carcinom zu bezeichnen, da dieser Begriff, wenn auch in erster Linie ein pathologisch-anatomi- 
scher, nach gerade doch auch, die Malignität betreffend, ein klinischer geworden ist, und wir ausser Stande sind, unseren Tumor direct den bösartigen Geschwiilsten zuzurechnen; nach einer brieflichen Mittheilung des Vaters unserer Patientin ist dieselbe seit der Enucleation, also neun Jahre hindurch vollkommen gesund, was allerdings nicht direct gegen die Malignität der Neubildung spricht. Von den in der Litteratur verzeichneten Geschwülsten ähnlicher Art, die unten kurz beschrieben werden sollen, war nur eine, die John Griffith veröffentlichte, mit Sicherheit bösartig, indem sechs Monate nach der Enucleation ein Recidiv in der Orbita auftrat und der Patient sechs Monate darnach, wohl Metastasen in inneren Organen (ein Sectionsbericht liegt allerdings nicht vor) erlag.

In den fünf übrigen Fällen hatte die Geschwulst, als zur Enucleation geschritten wurde, erst eine relativ geringe Ausdehnung erreicht, anch erfahren wir nichts über das weitere Schicksal der Patienten.

J. Michel war der erste, welcher im Jahre 1878 als eine Mischform zwischen Endo- und Epithelkrebs einen mässig pigmentirten Tumor beschrieb, welcher sich aus den Zellen der Pars eiliaris retinae entwickelt hatte. Es handelte sich um das rechte Ange einer 41jälrigen Frau. Die Geschwulst setzte sich aus einem zierlichen Maschenwerk sehmaler Bindegewebsbälkchen zusammen, in welches theils grössere und kleinere Zellplatten mit wechselnder Kernzall, theils schöne Cylinderzellen eingelagert waren, welche vollkommen den normalen der Pars ciliaris gliehen. Indem diese Zellen, zu beiden Seiten der Bindegewebsbälkchen angeordnet, innerhaib der Masche eine längsgestellte Lücke fiei liessen, entstand manchmal das Bild einer schlauchförmigen Drüse.

Die Beobachtung von Lagrange betriftt das linke Auge eines achtjährigen Knaben, welches schon drei Jahre vorher erblindet war. Der Ciliarkörper schloss zwei kleine Tumoren ein, deren histologische Untersuchung im wesentlichen drei verschiedene Arten Structur nachwies: 1. Zellschlänche, die ein centrales Lumen aufwiesen und von einer einfachen Lage Cylinderzellen ausgekleidet waren, 2. solehe, deren Lumen durch proliferirende Epithelzellen ausgefüllt war und 3. Massen von dicht neben ein- 
ander liegenden Epithelzellen; Lagrange lässt die Geselhwulst, die theils adenomatösen, theils carcinomatösen Bau zeigte, von den tubulösen Ciliarkörperdrüsen, welche Treacher Collins und Nicati beschrieben, ausgehen.

Treacher Collins untersuchte das linke Auge einer 28 jährigen Frau; er fand in demselben einen primären Tumor: des Ciliarkörpers, dessen Ursprung er in jene von ihm und Nicati beschriebenen tubulösen Drüsen verlegt. Die Geschwulst, welche in ihren vorderen $3 / 5$ stark pigmentirt, in ihrem hinteren Abschnitt pigmentfrei war, setzte sich aus Zellen von zweifellos epithelialem Typus zusammen und zeigte namentlich in den peripheren Theilen einen tubulösen Bau.

Griffit th theilt, wie bereits oben erwähnt, den ausführlichen Befund des linken Auges eines 37 jährigen Mannes mit, bei welehem die Diagnose auf Melanosarkom der Chorioidea gestellt worden war. Die Zellen, welche im Allgemeinen einen grossen Protoplasmaleib mit grossem, ovalem Kern hatten, wiesen im Uebrigen, sowohl Form als Pigmentirung betreffend, zallieiche Variationen anf. Sie waren an manchen Stellen in Schlauchform angeordnet, während sie an anderen dielhtgedrängt, ohne deutliche Intercellularsubstanz neben einander lagen. In dem vorderen Theil dieser epithelialen Neubildung, welche von den Zellen der Pars eiliaris retinae ausging, waren colloidälnnliche Massen eingestreut.

Dieser Tumor ähnelt in seinem histologisehen Bau am meisten dem unseren.

Pergens beschrieb als Adenom des Ciliarkörpers eine Geschwulst aus dem rechten Auge eines 56jährigen Mannes. Die Schnitte boten einen deutlich schlauchförmigen Charakter dar und ibre Zellen glichen auffallend den Epithelien der Ciliarregion.

Hanke fand in einem p. m. enucleirten Ange einer 65jährigen Fran als zufälligen Befund einen epithelialen Tumor des Ciliarkörpers, welcher von der inneren, umpigmentirten Epithellage der Ciliarfortsätze seinen Ursprung nahm. Die Neubildung ist ron einem Zellmantel, der directen Fortsetzung der retinalen Epithelreihen, umgeben, dessen imere Schicht Zellstränge abgiebt, die sich netzförmig verfleclten, und so ein System von verschieden grossen Hohlränmen umschliessen, welche mit ihren Wandungen die Hauptmasse des Tumors bilden. In den Strängen fliesst das Protoplasma der Zellen meist zu einer homogenen Masse zusammen, in welche die Kerne eingelagert sind. 
Zum Schlusse ist es mir eine angenehme Pflicht, Herrn Hofrath Prof. Fuchs für die Ueberlassung des Falles, sowie für das meiner Arbeit entgegengebrachte freundliche Interesse und die vielfache Unterstiitzung bei derselben, ergebenst zu danken.

\section{Literaturverzeichniss.}

J. Michel, Ueber Geschwülste des Uvealtractus. Y. Graefe's Arch. f. Ophthalm. XXIV. 1. S. 131.

Felix Lagrange, Carcinome primitif des procès et du corps ciliaire. Arch. d'Ophthalm. 1892. p. 143.

Treacher Collins, Descriptive Catalogue of Specimens in hosp. museum. Royal London Hosp. rep. XIV. 1. p. 14.

Treacher Collins, Transactions of ophthalm. Society. XIV. p. 83. Ophthalm. Rev. 1894. p. 38.

John Griffith, Transact. of ophthalm. Soc. XIV. p. 160. 1893-94. Pergens, Ueber Adenom des Ciliarkörpers als Ursache von Glaukom. Arch. f. Augenheilk. 1896. XXXII. S. 293.

V. Hanke, Zur Kenntniss der intraocularen Tumoren. v. Graefe's Arch. f. Ophthalm. XLVII. 3. p. 474.

Erklärung der Abbildungen auf Taf. XV, Fig. 1 u. 2.

Fig. 1. Uebergangsstelle der äusseren pigmentirten Zellreihe des Pigmentepithels in die Zellen der Geschwulst.

1 a. Dem Ciliarmuskel aufliegendes bindegewebiges Stroma.

1 b. Stark pigmentirte, der äusseren Zellreihe entstammende Zellen.

1. c. Uebergang derselben in die Zellen des Tumors unter Abnahme der Pigmentirung.

1 d. Polynucleärer Leukocyt.

1e. Mononucleäre Leukocyten.

Fi g. 2. Stelle des Tumors mit starker fettiger Degeneration der Geschwulstzellen. Die grossen blassen Kerne sind diejenigen der Tumorzellen. Hie und da Leukocyten. 
Fig.
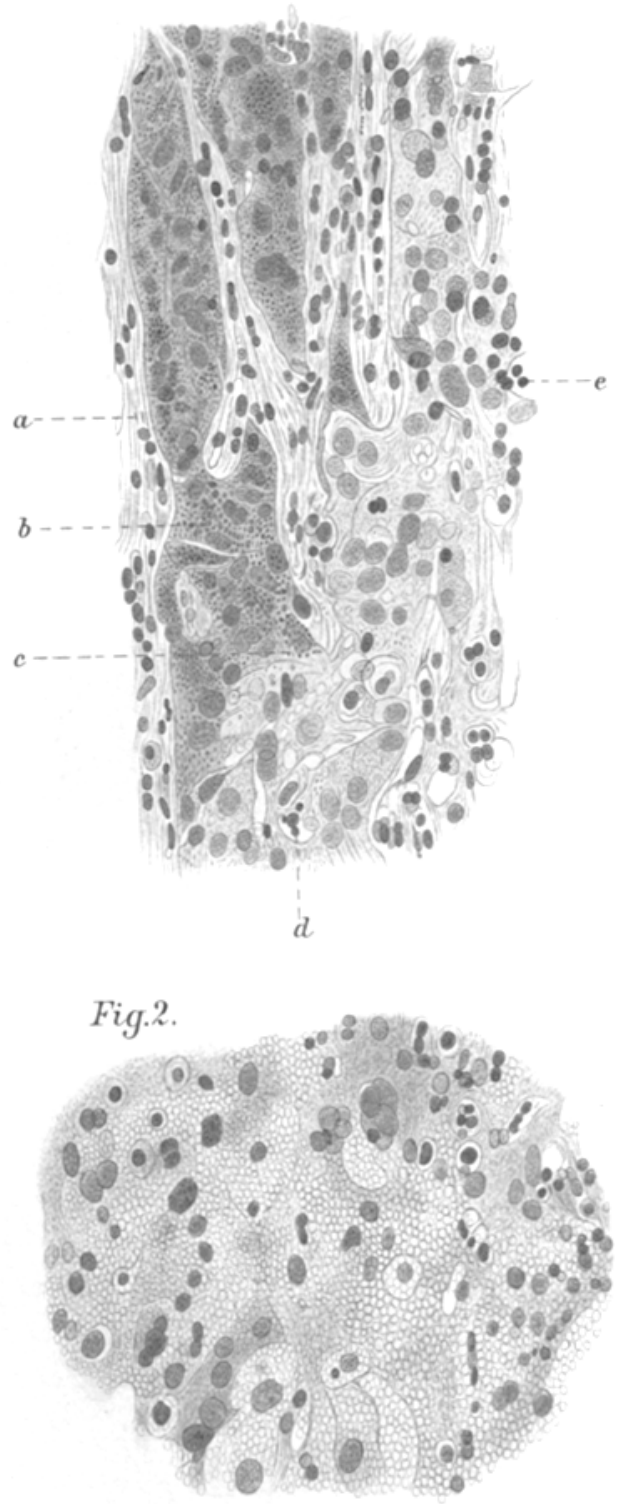

JWental del. 